\title{
Radiation detection from phase-locked serial dc SQUID arrays
}

\author{
Kaplunenko, V. K.; Mygind, Jesper; Pedersen, Niels Falsig; Ustinov, A. V.
}

Published in:

Journal of Applied Physics

Link to article, DOI:

$10.1063 / 1.353143$

Publication date:

1993

Document Version

Publisher's PDF, also known as Version of record

Link back to DTU Orbit

Citation (APA):

Kaplunenko, V. K., Mygind, J., Pedersen, N. F., \& Ustinov, A. V. (1993). Radiation detection from phase-locked serial dc SQUID arrays. Journal of Applied Physics, 73(4), 2019-2023. https://doi.org/10.1063/1.353143

\section{General rights}

Copyright and moral rights for the publications made accessible in the public portal are retained by the authors and/or other copyright owners and it is a condition of accessing publications that users recognise and abide by the legal requirements associated with these rights.

- Users may download and print one copy of any publication from the public portal for the purpose of private study or research.

- You may not further distribute the material or use it for any profit-making activity or commercial gain

- You may freely distribute the URL identifying the publication in the public portal

If you believe that this document breaches copyright please contact us providing details, and we will remove access to the work immediately and investigate your claim 


\title{
Radiation detection from phase-locked serial dc SQUID arrays
}

\author{
V. K. Kaplunenko, ${ }^{\text {a) }}$ J. Mygind, N. F. Pedersen, and A. V. Ustinov ${ }^{\text {b) }}$ \\ Physics Laboratory I, The Technical University of Denmark, DK-2800 Lyngby, Denmark
}

(Received 10 August 1992; accepted for publication 19 October 1992)

\begin{abstract}
We report on synchronous operation of series arrays of inductively coupled superconducting quantum interference devices (SQUIDs). Each array consisted of $N=3$ or 11 dc SQUIDs with common inductances providing a strong interaction between neighboring cells. Externally shunted $\left(\beta_{c} \approx 1\right)$ trilayer $\mathrm{Nb}-\mathrm{AlO}_{x}-\mathrm{Nb}$ Josephson junctions were used. Coherent radiation was detected in the frequency range $f=67-73 \mathrm{GHz}$ while the array voltage was $V_{N}=N f \Phi_{0}$ $\left(\Phi_{0}=2.07 \times 10^{-15} \mathrm{~V} \mathrm{~s}\right)$. The stability of the coherent state was influenced by the external dc magnetic field. The linewidth depended on the external loading. For small loading it was $N$ times smaller than expected for a single cell. The influence of the inductive coupling mechanism on the operation of discrete Josephson junction circuits and the similarity to the coupling in layered structures of long Josephson junctions is discussed.
\end{abstract}

\section{INTRODUCTION}

Coherent effects in arrays and other networks of Josephson junctions have been studied for several decades. ${ }^{1}$ For example in connection with granular films of high- $T_{c}$ superconductors the investigation of networks of coupled Josephson junctions have produced numerous new ideas and results. ${ }^{2}$ Many different and more complicated Josephson junction low- $T_{c}$ structures have appeared especially in the field of the rapid single flux quantum $\operatorname{logic}^{3}$ (RSFQL). For both areas the interaction between two inductively coupled superconducting quantum interference devices (SQUIDs) seems to be of crucial importance.

The inductive coupling has been investigated theoretically in order to build the quasi-digital voltage doubler, ${ }^{4}$ and later experiments have confirmed these ideas. ${ }^{5}$ The voltage doubler is based on the so-called asynchronous coupling between SQUID loops where a fluxon and an antifluxon are passing simultaneously through the interferometers.

This paper reports on numerical simulations and experimental investigations of another kind of coupling between interferometers-the synchronous one. Here two fluxons (instead of a fluxon and an antifluxon) are simultaneously excited in two inductively coupled SQUIDs. The same type of effects may also exist in multilayer Josephson structures which will be briefly considered in the last section.

\section{MAIN IDEA AND COMPUTER SIMULATIONS}

Figure 1(a) shows the equivalent circuit being investigated. It involves three SQUID loops formed by pairs of junctions $J_{1}-J_{2}, J_{3}-J_{4}$, and $J_{5}-J_{6}$, respectively. All these SQUIDs have the common inductances $L_{2}$ and $L_{3}$ which provide a strong coupling between them.

\footnotetext{
a) Permanent address: Institute of Radioengineering and Elcctronics, Russian Academy of Sciences, Moscow 103907, Russia.

b) Permanent address: Institute of Solid State Physics, Russian Academy of Sciences, Chernogolovka, Moscow district 142432, Russia.
}

The interaction mechanism may be explained as follows: If a fluxon appears in the interferometer $J_{1} L_{1} J_{2} L_{2}$ it causes a circular clockwise current around this contour. The phase difference over the inductance $L_{2}$ due to this current implies that some extra counter-clockwise current is excited in the $J_{3} L_{2} J_{4} L_{3}$ interferometer. This extra current adds to the bias current for the $J_{3}$ junction and may cause it to switch. This case is the asynchronous coupling as in the voltage doubler. ${ }^{4,5}$ For that kind of circuit such interaction appears because the bottom SQUID is continuously fed with flux quanta. In our case all the circuits are biased with the total currents $I_{1}, I_{2}$ and another kind of coupling should be considered. This may be understood from an energy point of view. When the three junctions $J_{1}, J_{3}$, and $J_{5}$ in the left arm are switched simultaneously and three fluxons are sitting in the corresponding interferometers, the total currents through the inductances $L_{2}$ and $L_{3}$ are minimized, giving rise to the configuration having the lowest energy for passing a bunch of flux quanta. The height of the energy barrier is proportional to the mutual inductance, however, the inductance should be small enough to prevent trapping of fluxons. The experimental circuits were designed with $L \approx \Phi_{0} / 2 I_{c}$ where $I_{c}$ is the critical current. For a more detailed description it should be possible to investigate analytically this type of circuit [Fig. 1 (a)] within the framework of the mutual phase locking technique. ${ }^{1}$

A numerical investigation gives some insight into the flux dynamics in the circuit. Figure 2 shows the time dependence of the instantaneous voltages at the nodes marked in Fig. 1(a) calculated with the actual parameters of the real circuit. Each voltage peak corresponds to a fluxon passing through each SQUID. Notice the rather good time coincidence of all peaks if no magnetic field is applied [Fig. 2(a)]. Thus the SQUIDs operate synchroneously and there will be very small currents in the inductances $L_{2}$ and $L_{3}$. If one applies a homogeneous magnetic field it influences only the inductances in the edge SQUIDs. Accordingly, introduction of additional currents with equal magnitude and opposite directions through the inductances $L_{1}$ and $L_{4}$ simulates the effect of the external 


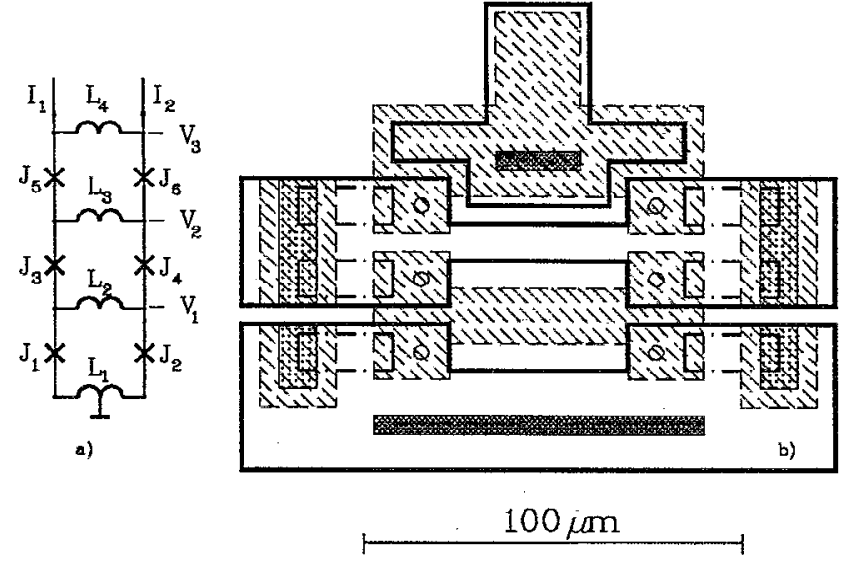

FIG. 1. Three series connected SQUIDs. (a) Equivalent circuit; $L_{1}=L_{2}$ $=L_{3}=L_{4}=7.8 \mathrm{pH}$. Identical shunted junctions with $I_{c}=82 \mu \mathrm{A}, R=1.9$ $\Omega$, and $C=0.7 \mathrm{pF}$ were used. (b) Actual layout; thick line-base electrode, dashed area-wiring, circles-Josephson junctions, dash point area-resistances, area with crosses-full connection between base and wire electrodes, cross-hatched area-contacts of base electrode with the ground plane.

magnetic field. Figure 2(b) shows the time dependent voltage when half a fiux quantum is penetrating into each edge SQUID. One can see that the field reduces the amplitude of the mutual oscillations and that some higher harmonics appear.
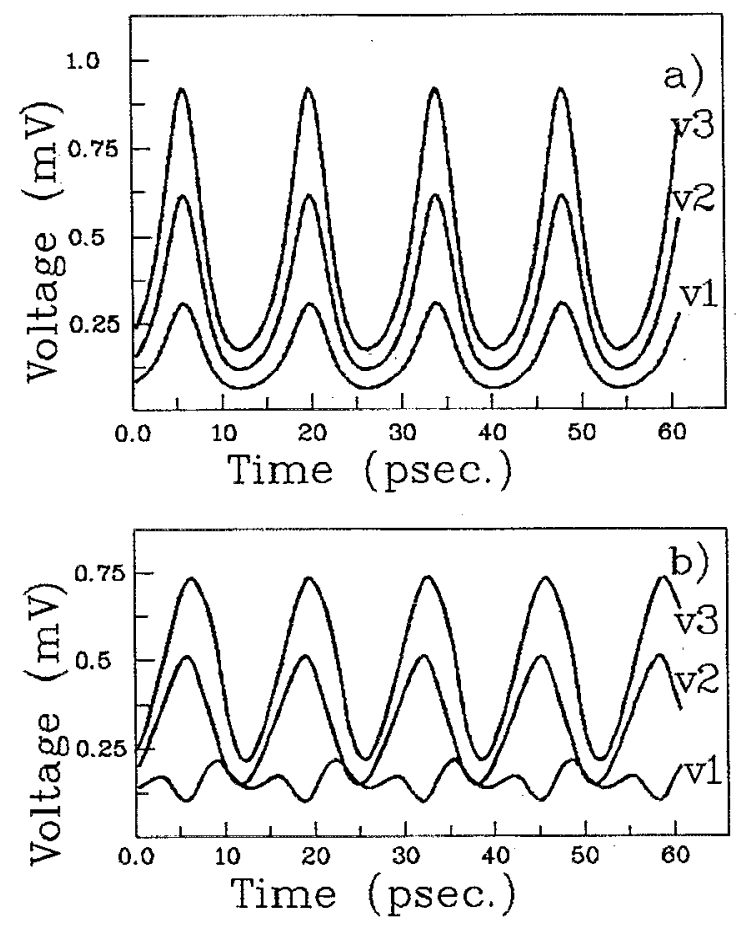

FIG. 2. Computer simulations of node voltages of the circuit depicted in Fig. 1(a). $I_{c}=107 \mu \mathrm{A}$. (a) without magnetic field, (b) with an applied magnetic field corresponding to half a flux quantum in the top and bottom SQUIDs.

\section{EXPERIMENTAL PROCEDURE}

The standard trilayer selective niobium etching and anodisation process (SNEAP) all-refractory technology has been employed to fabricate high quality $\mathrm{Nb}-\mathrm{AlO}_{x}-\mathrm{Nb}$ junctions with critical current densities of about $1 \mathrm{kA} / \mathrm{cm}^{2}$. The junctions were externally shunted in order to get an effective McCumber parameter $\beta_{c} \approx 1$. Figure 1(b) shows the layout of the three-SQUID array. All circuits are placed underneath the $\mathrm{Nb}$ ground plane. The top part of the circuit on Fig. 1(b) is via a microstrip transmission line connected to a finline antenna ${ }^{6}$ which transfers the emitted $\mathrm{mm}$-wave power from the array to the $60-90 \mathrm{GHz}$ rectangular waveguide leading to the room temperature receiver. The total length of the antenna structure on the substrate is $7.5 \mathrm{~mm}$ out of which the microstrip part is $1.8 \mathrm{~mm}$. Microwave current coming from the top electrode of the finline antenna flows in the ground plane, then "jumps" to the base electrode [cross-hatched area in Fig. 1(b)] and through the alternating base and wiring films returns to the other electrode of the antenna. The coupling inductances are defined by the various layers having different widths and magnetic gaps between the superconducting film and the ground plane.

The total dc resistance $R_{s}$ of the three-SQUID array was $2.8 \Omega$ and for the eleven-SQUID array it was $10.2 \Omega$. The differential resistance $R_{d}$ measured as the slope of the dc $I V$ curve of the three-SQUID array was of order 1-10 $\Omega$. The full width half-power linewidth $\Delta f$ of the emitted radiation may be estimated from

$$
\Delta f=\frac{4 \pi k_{B}}{\Phi_{0}^{2}} T \frac{R_{d}^{2}}{R_{s}}
$$

where $T$ is the Kelvin temperature and $k_{B}$ is Boltzmann's constant. Eq. (1) refers to the well known RSJ model with Nyquist type thermal noise (small fluctuations, low frequency cutoff). With the parameters stated the expected linewidths are as large as $2 \mathrm{GHz}$ at $4.2 \mathrm{~K}$.

For detection of signals with such large linewidths a standard $\mathrm{mm}$-wave spectrum analyzer with its harmonic mixer was not feasible. Instead a low-noise direct $\mathrm{mm}$-wave mixer powered at $f_{\mathrm{LO}}$ from a frequency locked Gunn diode local oscillator with was used. The output from the mixer was (if-)amplified and fed to a dc- $1.8 \mathrm{GHz}$ spectrum analyzer. The receiver therefore had two windows (sidebands) placed symmetrically with respect to $f_{\text {LO }}$. Typically $1 \mathrm{MHz}$ wide sidebands at $f_{\mathrm{LO}} \pm 20 \mathrm{MHz}$ were used. If the frequency distance between the sidebands was chosen to be larger than the linewidth of generation the observed signal as expected was split into two peaks of equal shape reflecting the action of the two sidebands. The double sideband (DSB) noise figure of the receiver in the band 68-72 $\mathrm{GHz}$ was less than $15 \mathrm{~dB}$ including the insertion loss of the waveguide leading to the room temperature mm-wave mixer and a series of isolators which prevented the local oscillator signal from reaching the array.

The substrate with its finline antenna was inserted in a slit cut symmetrically in the broad walls of the $60-90 \mathrm{GHz}$ waveguide. The external magnetic field was generated by a 


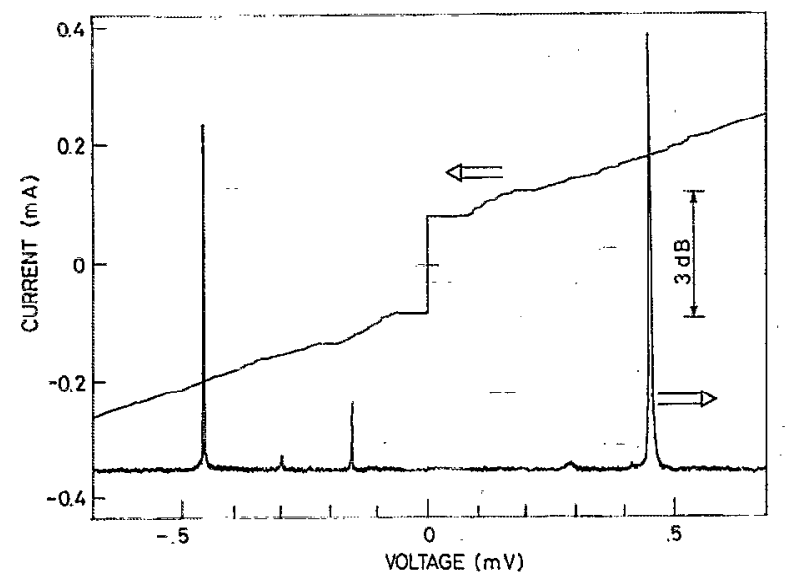

FIG. 3. de $I V$ curve of a three-SQUID array and the corresponding power of emitted mm-wave radiation detected in a receiver with two 1 $\mathrm{MHz}$ wide sidebands placed $\pm 20 \mathrm{MHz}$ from $f_{\mathrm{LO}}=70.75 \mathrm{GHz} . T=4.2$ $\mathrm{K}$.

2-mm-diam ring-shaped wire which was positioned under the sample in a distance of about $1 \mathrm{~mm}$. It was not possible to accurately calculate the value of this magnetic field because the ground plane significantly influences it near the junction areas. Accordingly, only relative units for the magnetic field proportional to the current in the ring are given.

The measurements were made at $4.2 \mathrm{~K}$ with the sample holder immersed directly in liquid helium. All leads used for dc current bias of the array and for generating the magnetic field were carefully twisted and electrically shielded. The sample holder was surrounded by a $\mu$-metal magnetic shield and the measurements were made in a rf-shielded room.

\section{EXPERIMENTAL RESULTS}

Figure 3 shows a typical $I V$ characteristic and the corresponding received $\mathrm{mm}$-wave power for a three-SQUID array. The $I V$ curve is very nonlinear but these peculiarities disappear together with the destruction of the coherent state when the external magnetic field is increased sufficiently. The results obtained were reproducible when changing the applied magnetic field. A small off-set field was needed in order to compensate for the nonuniform magnetic flux trapped in the niobium films. The trapped flux might also be the reason for the small difference in heights of the symmetrical radiation peaks (see Fig. 3). In accordance with the simulation results the application of the magnetic field reduces the radiation amplitude and excites higher harmonics. The small peak is observed at the voltage corresponding to the one third of the frequency of the main peak. This third harmonic generation is expected to be due to the time delay between the oscillations of different SQUIDs caused by the magnetic field [see Fig. 2(b)]. If microwave power with approximately the same frequency as the generated radiation is applied the Shapiro steps on the $I V$ curve directly show the position of all peaks.

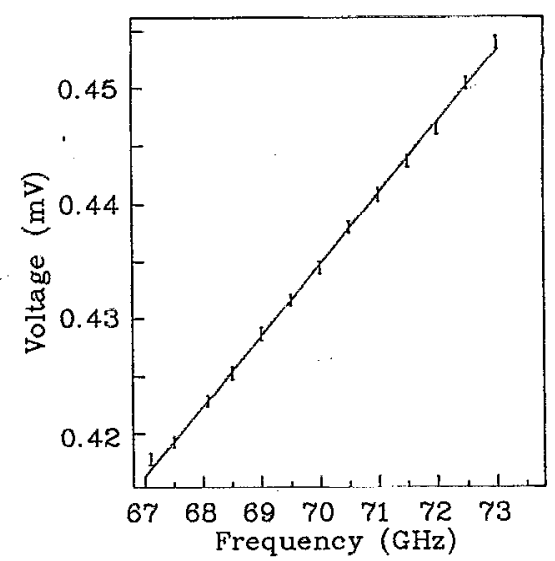

FIG. 4. Voltage as function of frequency of the detected radiation from a three-SQUID array. The full line represents a least-mean-square fit with slope $2.99 \times \Phi_{0}$.

Since the total voltage across the array is measured, the peaks of radiation appears at the voltages being three times larger than predicted by the fundamental Josephson relation $V=f \Phi_{0}$. Figure 4 shows the measured voltage position of the peak of radiation versus the detector center frequency. The slope of the full line obtained as a leastmean-square fit is $2.99 \times \Phi_{0}$. Within the experimental uncertainty this means that we have a coherent phase-locked state for the three-SQUID array obeying the modified Josephson relation $V_{N}=N f \Phi_{0}$ over a rather large frequency range.

The computer simulations (Sec. II) demonstrated that it is impossible to destroy the coherent state of the threeSQUID array with an external magnetic field. Since, however, the experimental magnetic field is somewhat nonuniform a complete detuning of the coupling is possible only at large magnetic fields [see Fig. 5(c)]. Moderate magnetic fields only reduce the amplitude of the emitted radiation. Unfortunately, in this experiment it is not possible to relate the power of radiation coming from the multi-SQUID coherent array [Fig. 5(a)] with the power from the individual SQUIDs [Fig. 5(c)] because the parameters, especially the differential resistance, of a single cell cannot be measured directly.

The coherent operation of the array can be verified by studying the linewidth of the emitted radiation. The results are depicted in Fig. 6 where the line with the steepest slope $170 \mathrm{MHz} / \Omega$ is calculated from Eq. (1) using the bath temperature $T=4.2 \mathrm{~K}$. The other line has a slope being three times smaller. Points mark the experimental results. It is evident that the three-SQUID array radiates in the coherent state, and that the linewidth of radiation is three times smaller than expected for the one-cell model. ${ }^{1}$ In Fig. 6 the small vertical shift of the experimental points seen at small linewidths may be due to excess noise in the measurement system. A few points indicate a larger deviation caused by the influence of parasitic magnetic fields. For large $R_{d}$ values the linewidth saturates and finally decreases as may be expected since the voltage interval cor- 


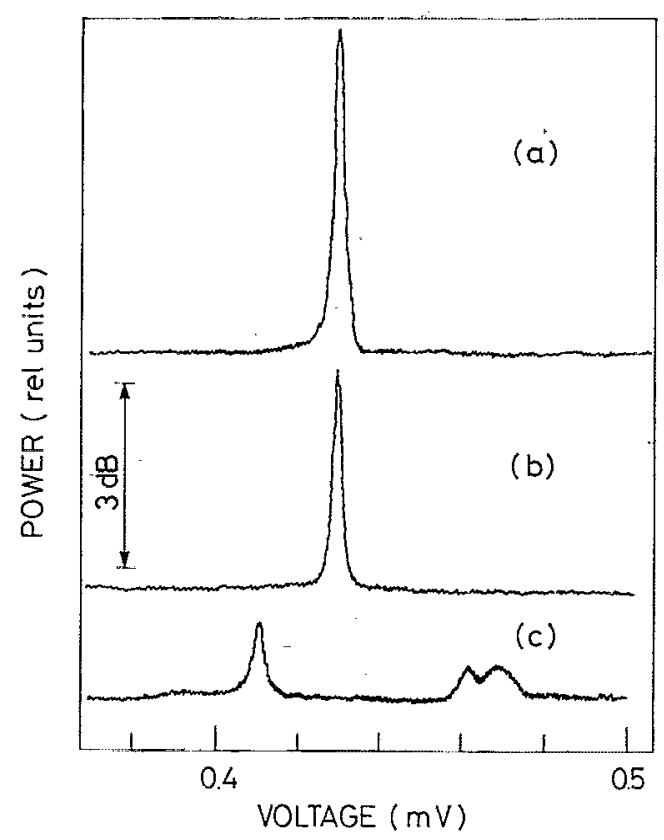

FIG. 5. Detected power vs voltage for a three-SQUID array in presence of an applied magnetic field $B$. (a) $B=0.0$, (b) $B=3.0$, and (c) $B=21$ in relative units. $F_{\mathrm{LO}}=69.5 \mathrm{GHz}$.

responding to the linewidth of generation significantly exceeds the voltage range where this resistance remains unchanged.

The important fact is that this type of series connection of $N$ phase-locked SQUIDs leaves the linewidth of the emitted radiation generation unchanged while the output resistance increases linearly with $N$. Accordingly the power of radiation delivered from this type of Josephson generators may be increased considerably being only limited by the impedance matching to the receiving network.

The above result has been also verified for ten coherently radiating SQUIDs placed on an eleven-cell array substrate. Unfortunately the missing (defect connection) cell made it difficult to do this with the same accuracy. The number of coherently working stages was inferred from the measured dc voltage and the frequency of the radiation

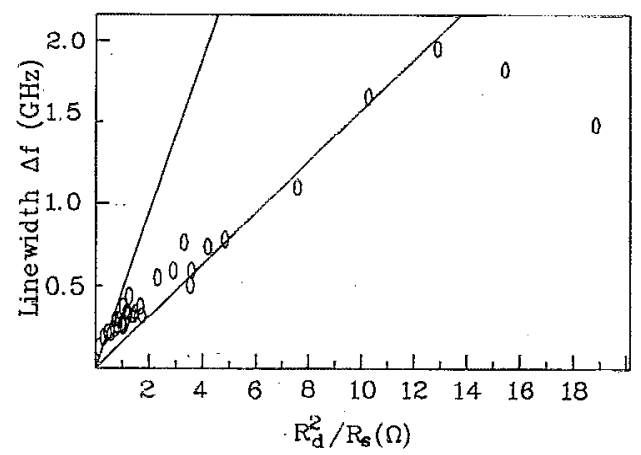

FIG. 6. The experimental full width half-power linewidth $\Delta f$ plotted vs $R_{d}^{2} / R_{s}$ for a three-SQUID array. The lines have the slopes of 170 and 57 $\mathrm{MHz} / \Omega$.
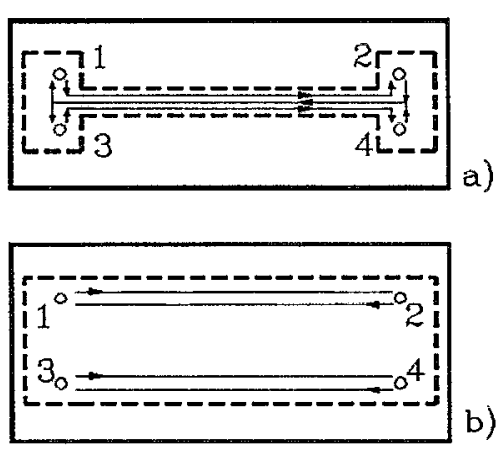

b)

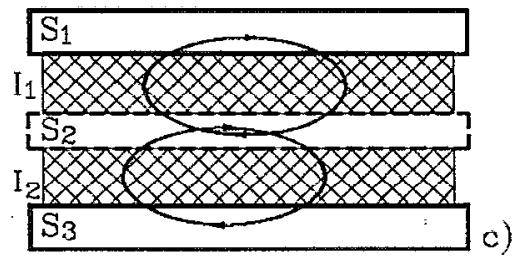

FIG. 7. Mutual interaction of two interferometers formed by junctions $J_{1}-J_{2}$ and $J_{3}-J_{4}$, respectively. The circles present the junction areas, the open box is the base electrode and the dashed lines restrict the wiring layer. (a) Strong coupling, (b) weak coupling, (c) multilayer $S-I-S-I-S$ Josephson junction equivalent.

peak using $V_{N}=N f \Phi_{0}$. The computer simulations of an eleven-SQUID array have shown that the coherent state depends on the initial conditions. If a flux quantum is placed near the center of such an array it splits the array into two coherent sections with a mutual phase difference between them. This might be another explaination for the fact that the coherent radiation could not be detected from any of the eleven-SQUID arrays tested.

\section{DISCUSSION AND CONCLUSIONS}

The effect of synchronous phase locking of a series array of dc SQUIDs has been demonstrated. The inductive coupling scheme used may have an important potential in other multijunction Josephson circuits.

The interaction between the SQUIDs may be qualitatively explained on the basis of the two layer structures shown in Figs. 7(a) and 7(b). For clarity, only the interaction between the two interferometers with junctions $J_{1}-J_{2}$ and $J_{3}-J_{4}$ is considered. The small circles are the junction areas between the base electrode (continuous line) and the wiring (dashed line). When the top film is shaped as a narrow bridgc [see Fig. 7(a)] the current flowing for both SQUIDs shares the same path, and hence, if some phase differences appears in the first interferometer it is directly applied to the other interferometer. If, on the other hand, the top film is very wide as in Fig. 7(b) the phase differences in one interferometer does not influence the other, corresponding to the situation where the distance between the interferometers becomes large.

Another case of inductively coupled Josephson junctions appears for multilayer structures. Figure $7(\mathrm{c})$ presents two long Josephson junctions $S_{1}-I_{1}-S_{2}$ and $S_{2}-I_{2}-S_{3}$ connected via a thin layer $S_{2}$ of a superconduc- 
tor. If the thickness of this layer is less than the London penetration depth, the same effect of interaction between two fluxons appears. ${ }^{7}$ A small thickness of this layer corresponds to a strong coupling of the SQUIDs [Fig. 7(a)] and a large one to the case of weak coupling [Fig. 7(b)]. Summarizing it seems possible to make an experimental discrete model of the interaction between layered structures of long Josephson junctions by employing the inductive mechanism of the series coupled SQUIDs. Such work is in progress. ${ }^{8}$

\section{ACKNOWLEDGMENTS}

The authors wish to thank O. V. Kaplunenko for fabricating the samples and V. P. Koshelets, Yu. Ya. Divin, and G. A. Ovsyannikov for helpful discussions. We also acknowledge the hospitality of Danish Institute of Fundamental Metrology, Lyngby where most of the mm-wave measurements were made. The work was partially supported by the Danish Natural Science Foundation (SNF), the Danish Research Academy (FA), and NATO (Grant No. LG-920672).

${ }^{1}$ A. K. Jain, K. K. Likharev, J. E. Lukens et al. Phys. Rep. 109, 309 (1984).

2See articles in: Proceedings of the NATO Advanced Research Workshop on Coherence in Superconducting Networks, edited by J. E. Mooij and G. Schön, Physica (North-Holland, Amsterdam, 1988), Vol. 152 B.

${ }^{3}$ K. K. Likharev and V. K. Semenov, IEEE Trans. Appl. Supercond. 1, 3 (1991).

${ }^{4}$ V. K. Kaplunenko, Sov. J. Supercond. 4, 629 (1991).

${ }^{5}$ V. K. Kaplunenko, V. P. Koshelets, M. I. Khabipov, V. M. Golomidov, and S. A. Kovtonyuk, Supercond. Sci. Technol. 4, 671 (1991).

${ }^{6}$ A. V. Ustinov, J. Mygind, and V. A. Oboznov, J. Appl. Phys. 72, 1203 (1992).

${ }^{7}$ S. Sakai, P. Bodin, and N. F. Pedersen (unpublished).

${ }^{8}$ V. K. Kaplunenko, J. Mygind, N. F. Pedersen, and A. V. Ustinov (to be published). 